\title{
Internet Access for Children's Online Schooling during the COVID-19 Pandemic and Parental Mental Health
}

The outbreak of the coronavirus disease 2019 (COVID-19) causing millions of people to be infected has posed major public health and governance challenges. This study evaluates the extent to which the unavailability of internet for children to learn online during the pandemic affects parental psychological wellbeing. We find that parents having no internet for their children to learn online during to the pandemic are 40.37, 47.22, 43.68, and 46.90 percentage points more likely to feel anxious, worried, displeased, and depressed every day. The study calls for the expansion of supports for children and families during the pandemic, especially for disproportionately affected communities. 